\title{
Vaccination with Trypanosoma rangeli modulates the profiles of immunoglobulins and IL-6 at local and systemic levels in the early phase of Trypanosoma cruzi experimental infection
}

\author{
Vanina Marini ${ }^{1,2}$, Edgardo Moretti $^{1,3}$, Daniela Bermejo ${ }^{4}$, Beatriz Basso ${ }^{1,3} /+$ \\ ${ }^{1}$ Laboratorio de la Coordinación Nacional de Control de Vectores, Córdoba, Argentina ²Cátedra de Inmunología, \\ Facultad de Ciencias Agropecuarias, Universidad Católica de Córdoba, Córdoba, Argentina ${ }^{3}$ Cátedra de Pediatría, \\ Servicio de Neonatología, Facultad de Ciencias Médicas ${ }^{4}$ Inmunología, Departamento de Bioquímica Clínica, \\ Facultad de Ciencias Químicas, Universidad Nacional de Córdoba, Córdoba, Argentina
}

In America, there are two species of Trypanosoma that can infect humans: Trypanosoma cruzi, which is responsible for Chagas disease and Trypanosoma rangeli, which is not pathogenic. We have developed a model of vaccination in mice with T. rangeli epimastigotes that protects against T. cruzi infection. The goal of this work was to study the pattern of specific immunoglobulins in the peritoneum (the site of infection) and in the sera of mice immunized with T. rangeli before and after challenge with T. cruzi. Additionally, we studied the effects triggered by antigenantibodies binding and the levels of key cytokines involved in the humoral response, such as IL-4, IL-5 and IL-6. The immunization triggered the production of antibodies reactive with T. cruzi in peritoneal fluid (PF) and in serum, mainly IgG1 and, to a lesser magnitude, IgG2. Only immunized mice developed specific IgG3 antibodies in their peritoneal cavities. Antibodies were able to bind to the surface of the parasites and agglutinate them. Among the cytokines studied, IL-6 was elevated in PF during early infection, with higher levels in non-immunized-infected mice. The results indicate that $\mathrm{T}$. rangeli vaccination against $\mathrm{T}$. cruzi infection triggers a high production of specific IgG isotypes in PF and sera before infection and modulates the levels of IL-6 in PF in the early periods of infection.

Key words: vaccination - Trypanosoma cruzi - Trypanosoma rangeli - IgG subclasses - interleukin 6 - peritoneal fluid

Trypanosoma cruzi is the etiologic agent of Chagas disease, a widespread infection in Latin America, which affects nearly 16 million people and leaves approximately 90 million people at risk of infection (Coura 2007). Non-specific immunosuppression that occurs in the acute phase of infection (Kierszenbaum et al. 1993, de Meis et al. 2009), in addition to the ability of T. cruzi to evade the immune response and adapt to the host environment, enables the parasite to invade cells and replicate in phagocytes, allowing parasite dissemination. Despite the fact that different research groups have proposed strategies to control parasite infection (Fontanella et al. 2008, Cazorla et al. 2009, Parodi et al. 2009, Rodrigues et al. 2009), as in other parasite diseases, there is not a fully effective vaccine available.

Our laboratory has developed an experimental vaccine model using Trypanosoma rangeli as an immunogen. $T$. cruzi and $T$. rangeli share endemic areas, reservoirs and vectors and show a high antigenic similarity (Basso et al. 1984, 1987, Cuba Cuba 1998). However, T. rangeli is not pathogenic in humans (Vallejo et al. 2007, 2009). Immunization with $T$. rangeli significantly

\footnotetext{
Financial support: Secretaría de Ciencia y Técnica, Universidad Nacional de Córdoba, Argentina, Coordinación Nacional de Control de Vectores, Argentina

+ Corresponding author: ebi@fcm.unc.edu.ar

Received 5 May 2010

Accepted 8 December 2010
}

reduces parasitemia in mice intraperitoneally infected with $T$. cruzi, enhances survival in the acute phase and does not induce autoimmune or histological alterations in the heart, spleen, skeletal muscle, intestine or other organs (Basso et al. 1991, 2008, Cervetta et al. 2003b). The immune response against complex structures of the parasites does not always produce a complete protective immunity. It has been shown that acute infection with T. cruzi induces a significant polyclonal activation of B cells contributing to the production of multiple antibody specificities that, in turn, can generate an autoreactive response (Minoprio 2001, Montes et al. 2006). However, antibodies play an essential role in the control of parasitemia and the generation of IgG1 and $\mathrm{IgG} 2$ antibodies are very important for parasite clearance (Brodskyn et al. 1989, Cordeiro et al. 2001).

The immune response to a microorganism is usually studied at the systemic level and little is known about the events that occur close to the site of infection. The aim of the present work was to study the immunological environment that $T$. cruzi will encounter when entering the peritoneum (the site of $T$. cruzi experimental infection) of vaccinated mice. We studied the expression profile of specific immunoglobulin isotypes in the peritoneum and in sera of mice immunized with $T$. rangeli before and after challenge with $T$. cruzi as well as the effects triggered by antigen-antibody binding. At the same time, the concentrations of IL-4, IL-5 and IL- 6 were analyzed in both compartments to discern a relationship between their production and the characteristics of the humoral response produced. 


\section{MATERIALS AND METHODS}

Parasites - T. cruzi: trypomastigotes of the Tulahuén strain were maintained by weekly intraperitoneal subinoculations in Balb/c mice. Blood samples of these animals were obtained by cardiac puncture 15 days post-infection (Pi). T. rangeli: the Colombian strain 2378 that was isolated and kindly provided by Dr Antonio D'Alessandro was cultured in monophasic medium (Basso et al. 1980). Epimastigotes were harvested in the exponential phase of growth, washed with phosphate buffer saline (PBS) and fixed with glutaraldehyde $(0.1 \%)$. They were washed with PBS again and resuspended in PBS at a concentration of $1 \times 10 \% / \mathrm{mL}$ (Basso et al. 1991).

Mice - Three-week-old Balb/c mice, both male and female, were maintained under standard conditions in the animal colony of the Division of Parasitology, Coordinación Nacional de Control de Vectores.

Vaccination and infection schedule - Groups of mice $(\mathrm{n}=6)$ were vaccinated with three doses of $0.1 \mathrm{~mL}$ of fixed T. rangeli epimastigotes emulsified in $0.1 \mathrm{~mL}$ of PBS containing $500 \mu \mathrm{g} / \mathrm{mL}$ of saponin (Sigma). Each mouse received $1 \times 10^{8}$ epimastigotes by subcutaneous injections 28, 21 and seven days prior to challenge with T. cruzi (day 0 of infection). The control group animals $(\mathrm{n}=6)$ were injected with PBS alone. One week after the last immunizing dose, all animals in both experimental groups received 1,500 trypomastigotes of $T$. cruzi by intraperitoneal infection. The levels of parasitemia were evaluated according to the Pizzi method (Cervetta et al. 2002) using $5 \mu \mathrm{L}$ of blood collected from the tail vein on days 10,15 and $20 \mathrm{Pi}$. For parasitemia and mortality evaluations of both groups, a total of 60 mice were examined. For immunological assays, mice were sacrificed by $\mathrm{CO}_{2}$ asphyxiation at different days $\mathrm{Pi}$ and peritoneal fluid and blood were obtained. Non-vaccinated and infected littermates were processed in parallel and are referred to as control mice in the text.

Peritoneal fluid (PF) and serum - Peritoneal cells and fluid from vaccinated and control mice were obtained by peritoneal washouts performed with $2.5 \mathrm{~mL}$ of Roswell Park Memorial Institute (RPMI) medium (Gibco, USA) plus $10 \%$ foetal bovine serum (FBS) (Natocor, Argentine). After $15 \mathrm{~min}$ of centrifugation at $300 \mathrm{~g}$, the supernatant (PF) was obtained and used for antibody and cytokine quantification. PF and sera were obtained before infection, during the first day after T. cruzi challenge as well as 15 and 20 days Pi.

Indirect haemagglutination (IHA) and indirect immunofluorescence (IIF) for total specific immunoglobulins - Red cells coated with total antigens of T. cruzi and obtained from a commercial kit were used for IHA following the instructions of the manufacturer (Wiener, Argentina).

In PF, the cut-off was a dilution of $1: 4$, taking into account the dilution in the technique's washes $(2.5 \mathrm{~mL}$ of RPMI). In serum, the cut-off was 1:8. For IIF, T. cruzi epimastigotes fixed with glutaraldehyde were incubated with sera or PF at $37^{\circ} \mathrm{C}$ for 30 min. Later, they were washed and incubated with fluorescein isothiocyanate total anti-mouse Igs (Biocientífica, Argentine) at $37^{\circ} \mathrm{C}$ for $30 \mathrm{~min}$, washed again and observed on an epifluores- cence microscope (Zeiss). Dilutions of PF in PBS higher than 1:8 were considered positive. In serum, a dilution of $1: 16$ was considered the cut-off. In both reactions, the results were expressed as base-2 logarithms of the titers.

Enzyme linked immunosorbent assay (ELISA) for immunoglobulin isotypes - T. cruzi-specific IgM, IgA, $\mathrm{IgE}$ and IgG isotypes were determined by ELISA (Sigma). The plates were coated with $30 \mathrm{ug} / \mathrm{mL}$ of $T$. cruzi antigens obtained from the supernatant of epimastigote (Tulahuén strain) homogenate centrifuged at $105,000 \mathrm{~g}$ (F105) (Cervetta et al. 1998, Montes et al. 2006). Sera and $\mathrm{PF}$ were considered positive if the mean optical density (OD) value measured at $450 \mathrm{~nm}$ in an ELISA plate reader was two standard deviations above the mean value for control samples assayed in parallel. The results were expressed as the mean of the OD values \pm standard error.

Parasite agglutination and immunofluorescence test - T. cruzi epimastigotes and trypomastigotes were incubated in culture plates with $250 \mu \mathrm{L}$ of serial dilutions of $\mathrm{PF}$ or serum of both experimental groups (up to $1 / 128$ ) in RPMI with PBS at $37^{\circ} \mathrm{C}$ and $5 \% \mathrm{CO}_{2}$ Parasites incubated with RPMI containing $10 \%$ PBS were used as control. After $3 \mathrm{~h}$, each reservoir was observed under a 400X optical microscope. Clustering of four or more associated epimastigotes or trypomastigotes was considered agglutination. Additionally, glutaraldehyde-fixed parasites were incubated with both material from vaccinated and control mice under the aforementioned conditions for the immunofluorescence reaction and they were observed using an epifluorescence microscope.

Determination of $I L-4, I L-5$ and $I L-6$ by enzyme immunoassay - ELISA was used for the quantification of IL-4, IL-5 and IL-6 (BD, USA) in PF. Plates coated with a monoclonal antibody against the studied cytokine were incubated with the samples and standards. After the plates were washed, a peroxidase-conjugated antiserum specific to each cytokine was added and the plates were again incubated and washed. Next, peroxidase-conjugated streptavidin was added and the colour reaction was developed with tetramethyl-benzidine following a final cycle of incubation and washing. After adding $\mathrm{H}_{2} \mathrm{SO}_{4}$ to halt the colour reaction, absorbencies were measured at $450 \mathrm{~nm}$ in an ELISA plate reader. Values were calculated from standard curves based on dilutions of recombinant cytokines. The assay was sensitive to lower limits of $179 \mathrm{pg} / \mathrm{mL}$ for IL-4 and IL-5 and $4 \mathrm{pg} / \mathrm{mL}$ for IL-6.

Ethical standards - All experiments reported herein were conducted in compliance with the Animal Welfare Act and in accordance with the principles set forth in the Guide for the Care and Use of Laboratory Animals (Institute of Laboratory Animal Resources, National Research Council, National Academy Press, 1996). Mice were cared for according to the ethical standards for animal testing and experimentation. The institutional number of the license to work with mice infected with $T$. cruzi is $122 / 08$.

Statistical data analysis - Student $t$ test was used to determine differences between mean values of the different groups. The level below which statistical significance was considered to exist was $p<0.05$. 


\section{RESULTS}

T. rangeli vaccinated mice present lower levels of parasitemia than control mice - Fig. 1 shows a representative experiment of the parasitemia levels in mice immunized with $T$. rangeli and infected with $T$. cruzi and their respective controls. Fifteen days $\mathrm{Pi}$, levels of circulating trypomastigotes in vaccinated animals were significantly lower than in unvaccinated animals. Furthermore, parasitemia continued to increase in control animals, showing statistically significant differences in both groups $(\mathrm{p}<0.05)$. Twenty days after infection, the mortality rate in the control group was approximately $95 \%$ and in vaccinated mice, it was $5 \%$.

T. rangeli immunization induces $T$. cruzi-cross reactive antibodies in PF - Fig. 2 shows that the immunization induces high titers of antibodies to T. cruzi in PF, which remain similar after and during infection. The group of non-immunized-infected animals showed an increase in antibodies as a typical response to infection, although at lower titers compared to those in the group of vaccinated animals. Similar results were obtained when blood trypomastigotes forms were employed as antigen in immunofluorescence test (data not shown). Values obtained from sera samples of both groups of animals showed similar results compared to PF. Results obtained in sera were in agreement with those seen in a previous work (Basso et al. 1991).

T. rangeli vaccination triggers high production of parasite-specific immunoglobulin isotypes in PF and sera - As shown in Fig. 3, T. rangeli vaccination induced a significant increase in $\operatorname{IgG}$ isotypes in PF and sera, mainly IgG1, IgG2a and IgG2b, reaching levels that were maintained with slight variations in samples from 15 and 20 days Pi. Again, the group of non-immunized infected animals showed an increase in IgG isotypes in response to infection. Before infection, IgG3 was significantly higher in PF samples but not in sera from vaccinated

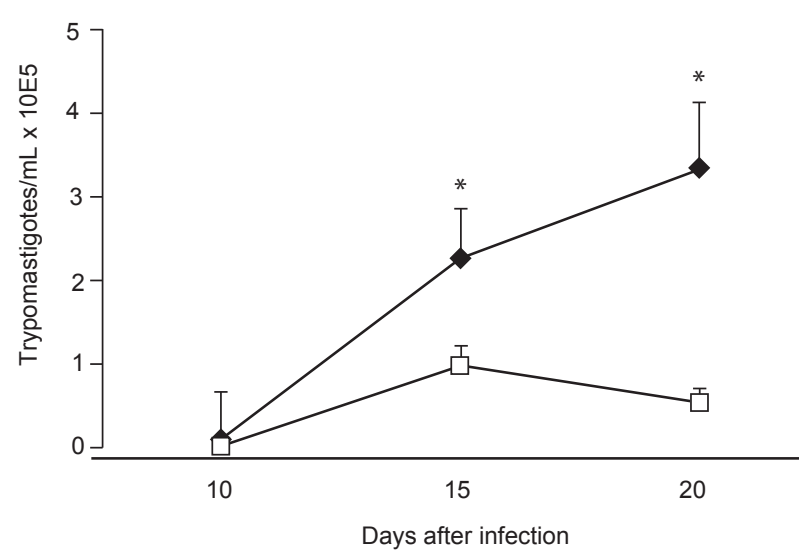

Fig. 1: parasitemia levels (geometric mean \pm standard error) in mice vaccinated with fixed epimastigotes of Trypanosoma rangeli $(\mathrm{n}=6)$ $(\square)$ or controls infected with Trypanosoma cruzi $(\mathrm{n}=6)(\diamond)$. Asterisk means significant differences between both groups evaluated by Student $t$ test $(\mathrm{p} \leq 0.05)$. mice when compared to control mice. Interestingly, IgG3 anti-parasite is not induced by $T$. cruzi infection and parasite-specific antibodies are not detected in PF or sera of non-vaccinated-infected mice.

With respect to the other isotypes in sera, the levels of IgM expressed in OD values were as follows: before infection: $\mathrm{C}: 0.19 \pm 0.07, \mathrm{~V}: 0.45 \pm 0.17(\mathrm{p}<0.05) ; 15$ days Pi: C: $0.81 \pm 0.07, V: 1.19 \pm 0.13(p<0.05) ; 20$ days Pi: C: $0.91 \pm 0.04, \mathrm{~V}: 1.04 \pm 0.09$. Similar results were obtained in PF (data not shown). The OD values of $\mathrm{IgE}$ obtained were as follows: before infection: C: not detected, V: 0.13 $\pm 0.02(\mathrm{p}<0.05) ; 15$ days Pi: $\mathrm{C}$ : not detected, $\mathrm{V}: 0.13 \pm$ $0.02(\mathrm{p}<0.05) ; 20$ days Pi: C: $0.01 \pm 0.06, \mathrm{~V}: 0.14 \pm 0.02$ $(\mathrm{p}<0.05)$. In this study, we were unable to detect any IgA. Prior to comparing the results obtained from both groups of mice, we performed serial dilutions of samples. The results obtained were identical to those performed with OD values shown above (data not shown).

Antibodies present in PF and sera bind to parasite surface - To test the ability of antibodies present in both compartments to recognize the parasite, T. cruzi epimastigotes and trypomastigotes were incubated with PF or serum from immunized and control mice for $3 \mathrm{~h}$. Agglutination and decreased mobility of parasites was observed up to a 1/32 dilution of PF obtained from immunized mice. Additionally, $70 \%$ of epimastigotes and trypomastigotes agglutinated in PF from vaccinated mice (Fig. 4A1, 2). Sera provided the highest percentage,
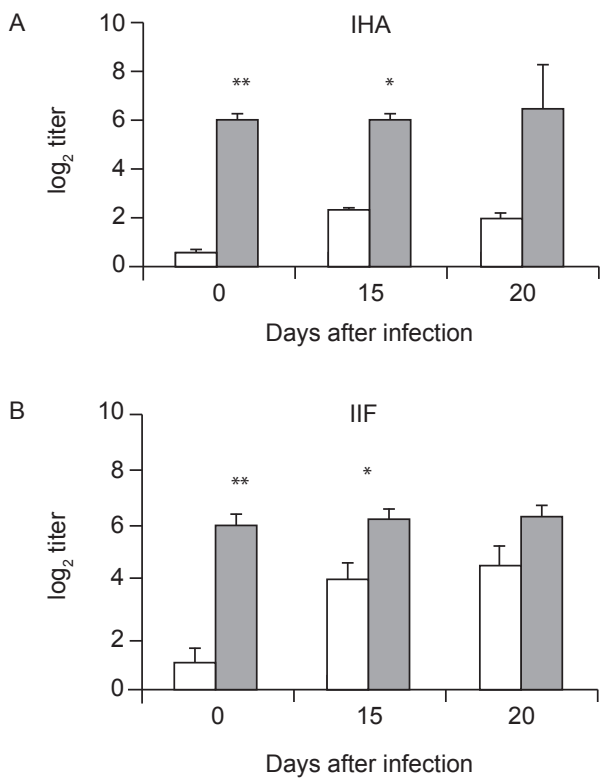

Fig. 2: specific antibodies levels $\left(\log _{2}\right.$ mean \pm standard error) in peritoneal fluid in mice vaccinated $(n=6)$ (grey bars) or not $(n=6)$ (white bars) with fixed epimastigotes of Trypanosoma rangeli before and after infection with Trypanosoma cruzi. A: indirect hemagglutination (IHA); B: indirect immunofluorescence (IIF). Asterisks mean significant differences between both groups evaluated by Student $t$ test $(*: \mathrm{p}$ $\leq 0.05 ; * *: \mathrm{p} \leq 0.005)$. 
near $100 \%$, whereas no clusters were observed in the presence of $\mathrm{PF}$ and sera from non-vaccinated-infected control mice or culture medium (Fig. 4B1, 2).

Parasite agglutination can be triggered by different molecules (e.g., lectins and antibodies) present in PF and sera from immunized mice; therefore, we investigated the presence of antibodies on parasite surfaces using immunofluorescence. Fig. 4Cl shows that agglutinated epimastigotes have specific antibodies on their surface indicating that these anti-parasitic antibodies mediated agglutination. As expected, parasites incubated with PF from control mice do not show fixed antibodies on their surface (Fig. 4C2). Similar results were observed with trypomastigotes (data not shown).

T. rangeli vaccination modulates the levels of IL-6 in $P F$ - Finally, we examined cytokines involved in plasma cell differentiation, key players in the outcome of an antibody response. Surprisingly, no IL-4 or IL-5 was detected with our assay. Nevertheless, mice vaccinated with $T$. rangeli and infected with $T$. cruzi display a significantly lower concentration of IL-6 in comparison to non-vaccinated-infected control mice. As shown in Table, the levels prior to infection were undetectable and all other IL-6 quantified levels ( $24 \mathrm{~h}, 15$ and 20 days $\mathrm{Pi}$ ) showed significantly lower concentrations in vaccinated mice $(\mathrm{p}<0.05)$.

\section{DISCUSSION}

Previous studies in our laboratory revealed that mice immunized with $T$. rangeli-fixed epimastigotes presented a substantial protection against infection with $T$. cru-

\section{TABLE}

Kinetic of IL-6 cytokine levels in mice peritoneal fluid at different post-infection (Pi) days

\begin{tabular}{lcccc}
\hline PI days & 0 & 1 & 15 & 20 \\
\hline Groups $(\mathrm{n}=6)$ & & & & \\
Controls & $\mathrm{ND}^{a}$ & $53.6^{b} \pm 6.9^{c}$ & $925.5 \pm 130.9^{c}$ & $78.0 \pm 12.5^{c}$ \\
Vaccinated & $\mathrm{ND}$ & $33.9 \pm 5.0$ & $524.8 \pm 20.8$ & $27.8 \pm 6.4$
\end{tabular}

$a$ : the results of optical density were below the detection capacity of the assay $(<4 \mathrm{pg} / \mathrm{mL}) ; b$ : $\mathrm{pg} / \mathrm{mL} ; c$ : $\mathrm{p}<0.05$. The differences in cytokine levels between controls vs. vaccinated were evaluated by Student $t$ test $(\mathrm{p}<0.05)$. ND: not detected.

$z i$, accompanied by a balance in Th1 and Th 2 cytokine production. Indeed, sera of immunized mice contained high concentrations of IFN- $\gamma$, IL-12p40, nitric oxide and soluble tumor necrosis factor receptor- $\alpha$ (TNFR- $\alpha$ ). Very low concentrations of IL-10 and modulation in the levels of IL-18, IL-6, TNFR- $\alpha$ were also detected (Basso et al. 1991, 2004, Cervetta et al. 2002, 2003a).

However, we also demonstrated that mice inoculated with different adjuvants presented a similar course of infection and cytokine pattern compared to mice in the control group that were treated only with PBS. As shown previously (Basso et al. 1991, Cervetta et al. 2002), as well as in this study, parasitemia levels in vaccinated mice were significantly reduced when compared to the parasite load in unvaccinated animals.
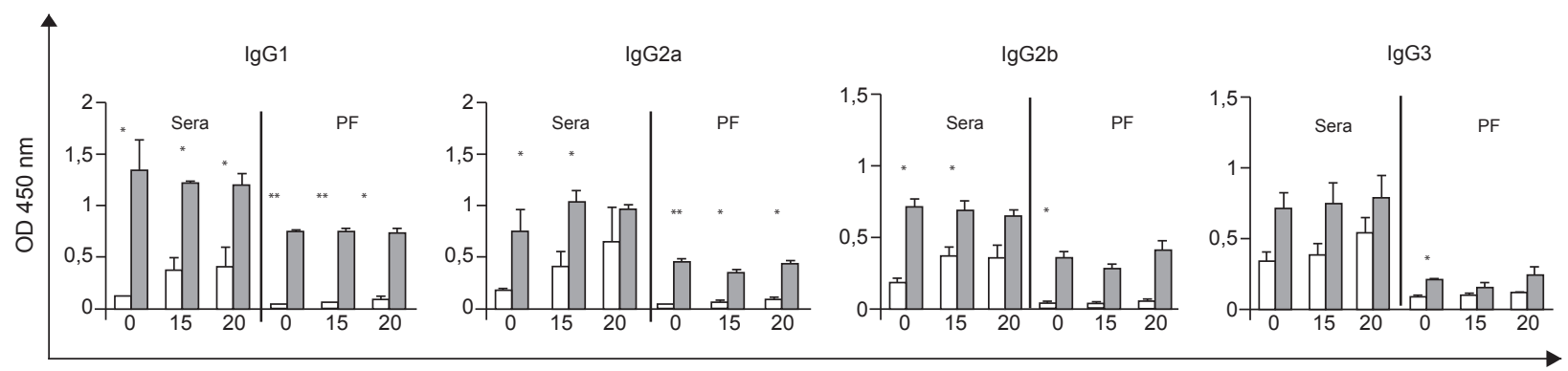

Days post infection

Fig. 3: specific IgG isotype levels in sera and peritoneal fluid (PF) obtained from vaccinated $(n=6)$ (grey bars) or control mice ( $n=6)$ (white bars) before and after infection determined by enzyme linked immunosorbent assay [optical density (OD) mean \pm standard error]. Asterisks mean significant differences between both groups evaluated by Student $t$ test $(*: \mathrm{p} \leq 0.05 ; * * \mathrm{p} \leq 0.005)$.

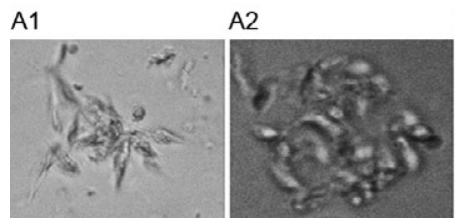

B1

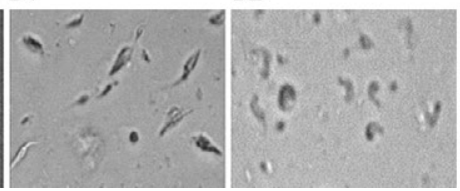

C1

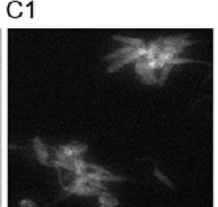

$\mathrm{C} 2$

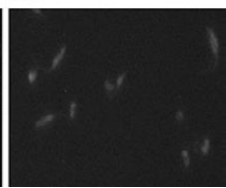

Fig. 4: parasite agglutination test (3 animals/group). Agglutination of Trypanosoma cruzi epimastigotes (A1) and trypomastigotes (A2) when they were incubated with peritoneal fluid (PF) from vaccinated mice. B1 and B2 shown no agglutination of $T$. cruzi epimastigotes and trypomastigotes, respectively, when they were incubated with PF from control mice (400X). Indirect immunofluorescence test (3 mice/group). T. cruzi epimastigotes were incubated with PF from vaccinated (C1) or control mice (C2) (400X). In all experiments the pictures show a representative field for each group. 
Because vaccination with $T$. rangeli triggers an important immune response against T. cruzi, the aim of the current study was to compare the profiles of specific immunoglobulin isotypes in PF and serum starting early in infection periods. We show that the clearance of circulating parasites in vaccinated mice could be associated with high titers of antibodies in PF and serum induced by immunization, suggesting that these may act as one of the early infection-control mechanisms. Given that different IgG subclasses are related to different immune response patterns, an examination of these immunoglobulins revealed high concentrations of IgG1 and IgG2 in the PF of vaccinated mice. These immunoglobulins are important effectors of the immune response and enhance parasite clearance rates (Brodskyn et al. 1989). A similar result was found at systemic levels in vaccinated mice, although with fewer oscillations. In the control group, the titers of IgG isotypes increased in parallel with the progress of infection. In this model, vaccination-induced parasite antibodies are present at high levels in the peritoneal cavity (the site of infection) prior to challenge with T. cruzi.

These increases in $\mathrm{IgG}$ isotypes are in agreement with a previous work in which we demonstrated that the immunization of mice with $T$. cruzi exoantigens was capable of protecting mice from a challenge with this parasite and was associated with high concentrations of IgG1 and $\mathrm{IgG} 2$ and low concentrations of $\mathrm{IgG} 3$ at the systemic level (Gruppi et al. 1991). Prior to infection, immunized mice maintain parasite-specific IgG3 antibodies only in the peritoneal cavity, suggesting a differential pattern of response in both compartments.

Other authors studying the acute period of T. cruzi infection detected that upon increases in serum levels of $\operatorname{IgM}, \operatorname{IgG}$ and IgG1, the parasite load decreased (Carneiro et al. 2007, Coura-Vital et al. 2008). In our work IgM, which is responsible for the primary specific immune response, was elevated in vaccinated and control animals in both biological fluids, but a higher concentration was always observed in vaccinated animals.

The protective role of the antibodies in the acute phase of the infection is dependent mostly on their ability to induce removal of trypomastigotes from the circulation, in addition to other concomitant cell-mediated events (Umekita \& Mota 2000). It has been demonstrated that parasite-specific $\operatorname{IgG}$, especially $\operatorname{IgG} 2$, recognizes a large number of parasite antigens and forms microaggregates that can fix complement, enhance opsonisation and mediate cytotoxicity mechanisms (Takehara \& Mota 1991). In this context, the agglutination and decreased parasite mobility observed when $T$. cruzi epimastigotes and trypomastigotes were incubated with both PF and serum of vaccinated mice suggest that the antigenic similarity between T. cruzi and T. rangeli (Basso et al. 1987) would lead to a humoral response. This response could be involved in the early elimination of the parasite through agglutination or lysis.

The increased systemic levels of the aforementioned immunoglobulin isotypes were maintained in vaccinated animals for at least nine months and they continued their protective role upon challenge with virulent $T$. cru$z i$ trypomastigotes (data not shown). Another immuno- globulin associated with effector functions is IgE which, when bound to surface receptors of immune system cells, induces inflammatory reactions and cytotoxicity mechanisms responsible for the elimination of parasites (Capron et al. 1992). The vaccinated animals in this study showed significantly increased levels of IgE before and after infection, in agreement with previous work (Gruppi et al. 1990, Basso et al. 1991), in which increased IgE levels were observed in mice vaccinated against exoantigens of $T$. cruzi and T. rangeli epimastigotes.

In relation to the production of cytokines possibly involved in the development of anti-parasite humoral response and its implication in the outcome of T. cruzi infection, it is known that cytokines produced by both subpopulations of helper T-cells are necessary for an adequate immune response (Capron et al. 1992). With the methodology applied in this study, neither IL-4 nor IL-5 were detected in PF or serum. Other authors also failed to detect these interleukins in supernatants of $T$. cruzi antigen-stimulated spleen cells (Tarleton 1991). The findings are consistent with results from our previous report (Cervetta et al. 2002) and do not preclude that a high production of these cytokines in secondary lymphoid tissues occurs. As previously described by other research groups using different immunization models (Tarleton 1991, Hoft 1993), it is possible that these soluble mediators might be increased in periods subsequent to those studied here. Interestingly, in the beginning of infection, IL-6 production in PF was down-regulated by vaccination, probably as a consequence of a minor parasite load. Nevertheless, non-immunized-infected mice revealed a significant increase in this cytokine following the early phase of the infection, which could be involved in tissue damage. Similar results were observed in a previous work at the systemic level (Cervetta et al. 2002), but we demonstrated here that this cytokine was detectable earlier in PF than in sera, suggesting again, differential patterns of response in both compartments. Additionally, the results suggest that the amounts of IL-6 produced in vaccinated animals were sufficient for lymphocyte differentiation; however, proinflammatory synthesis predominated in unvaccinated mice.

In conclusion, we compared for the first time the profile of immunoglobulin isotypes and IL-6 levels in the PF (site of challenge with T. cruzi) and at the systemic level, before and in the early period of infection in a $T$. rangeli vaccination model. Taken together, these results could account for some microbicide mechanisms that assist in the clearance of circulating trypomastigotes; these findings could then be extrapolated to other mucosa and cavities. It would be of interest to continue this study to encompass other mediators of the immune system in order to better elucidate the early mechanisms involved in the protection against $T$. cruzi infection.

\section{ACKNOWLEDGEMENTS}

To the Laboratoire de Parasitologie de la Faculté de Médecine, Université Libre de Bruxeles, who kindly provided us with some reagents used in this study, to Dr Adriana Gruppi, for her suggestions, to María Fernanda Garstein, Renata Defago and Ianina Moretti Basso, for their contribution to the translation of this paper. 


\section{REFERENCES}

Basso B, Cervetta L, Moretti ER, Carlier Y, Truyens C 2004. Acute Trypanosoma cruzi infection: IL-12, IL-18, TNF, sTNFR and NO in . rangeli-vaccinated mice. Vaccine 22: 1868-1872.

Basso B, Moretti E, Fretes R 2008. Vaccination with epimastigotes of different strains of Trypanosoma rangeli protects mice against Trypanosoma cruzi infection. Mem Inst Oswaldo Cruz 103: 370-374.

Basso B, Moretti ER, Domínguez M 1980. Cultivo de T. cruzi en medio monofásico con aireación para la obtención de antígenos. Medicina (Buenos Aires) 4: 428-432.

Basso B, Moretti ER, Vottero-Cima E 1984. Comportamiento antigénico del Trypanosoma (Schizotrypanum) cruzi y del Trypanosoma (Herpetosoma) rangeli frente a sueros de pacientes con enfermedad de Chagas. Medicina (Buenos Aires) 44: 475-479.

Basso B, Moretti ER, Vottero-Cima E 1987. Antigenic relationships between Trypanosoma cruzi and Trypanosoma rangeli. Rev Iber Parasitol 47: 15-21.

Basso B, Moretti ER, Vottero-Cima E 1991. Immune response and Trypanosoma cruzi infection in Trypanosoma rangeli-immunized mice. Am J Trop Med Hyg 44: 413-419.

Brodskyn CI, Silva AM, Takehara HA, Mota I 1989. IgG subclasses responsible for immune clearence in mice infected with Trypanosoma cruzi. Immunol Cell Biol 67: 343-348.

Capron A, Dessaint JP, Capron M, Pierce RJ 1992. Vaccine strategies against schistosomiasis. Mem Inst Oswaldo Cruz 87 (Suppl. IV): 19-27.

Carneiro CM, Martins-Filho OA, Reis AB, Veloso VM, Aráujo FM, Bahia MT, de Lana M, Machado-Coelho GL, Gazzinelli G, Correa-Oliveira R, Tafuri WL 2007. Differential impact of metacyclic and blood trypomastigotes on parasitological, serological and phenotypic features triggered during acute Trypanosoma cruzi infection in dogs. Acta Trop 101: 120-129.

Cazorla SI, Frank FM, Malchiodi EL 2009. Vaccination approaches against Trypanosoma cruzi infection. Expert Rev Vaccines 8: 921-935.

Cervetta L, Moretti E, Basso B 2002. Experimental Chagas' disease: the protection induced by immunization with Trypanosoma rangeli is associated with down-regulation of IL-6, TNF- $\alpha$, and IL10 synthesis. Acta Parasitol 47: 73-78.

Cervetta L, Moretti E, Basso B 2003a. Balance entre TNF y sus receptores solubles en la infección chagásica humana y experimental. Rev Alergia e Inmunologia Clinica 20: 61-66.

Cervetta L, Moretti E, Basso B, Castro I, Santamarina N 1998. Enfermedad de Chagas crónica: efectos del tratamiento en los niveles de anticuerpos hacia antígenos crudos y semipurificados del Trypanosoma cruzi. Bol Chil Parasitol 53: 3-9.

Cervetta L, Moretti E, Fretes R, Basso B 2003b. Vaccination with live epimastigotes of Trypanosoma rangeli protects mice against Trypanosoma cruzi lethal infection. Rev Patol Trop 32: 223-234.

Cordeiro FD, Martins-Filho OA, Da Costa Rocha MO, Adad SJ, Corrêa-Oliveira R, Romanha AJ 2001. Anti-Trypanosoma cruzi immunoglobulin G1 can be a useful tool for diagnosis and prognosis of human Chagas' disease. Clin Diagn Lab Immunol 8: 112-118.

Coura JR 2007. Chagas disease: what is known and what is needed - A background article. Mem Inst Oswaldo Cruz 102 (Suppl. I): 113-122.

Coura-Vital W, Carneiro CM, Martins HR, de Lana M, Veloso VM, Teixeira-Carvalho A, Bahia MT, Corrêa-Oliveira R, MartinsFilho OA, Tafuri WL, Reis AB 2008. Trypanosoma cruzi: immu- noglobulin isotype profiles during the acute phase of canine experimental infection with metacyclic or blood trypomastigotes. Exp Parasitol 120: 269-274.

Cuba Cuba A 1998. Review of the biologic and diagnostic aspects of Trypanosoma (Herpetosoma) rangeli. Rev Soc Bras Med Trop 31: 207-220.

de Meis J, Morrot A, Farias-de-Oliveira DA, Villa-Verde DM, Savino W 2009. Differential regional immune response in Chagas disease. PLoS Negl Trop Dis 3: e417.

Fontanella GH, De Vusser K, Laroy W, Daurelio L, Nocito AL, Revelli S, Contreras R 2008. Immunization with an engineered mutant trans-sialidase highly protects mice from experimental Trypanosoma cruzi infection: a vaccine candidate. Vaccine 26: 2322-2334.

Gruppi A, Pistoresi-Palencia MC, Basso B, Vottero-Cima E 1990. Mouse IgE response against exoantigens of Trypanosoma cruzi. Int Arch Allergy Appl Immunol 92: 56-59.

Gruppi A, Pistoresi-Palencia MC, Cerban F, Vottero-Cima E 1991. Trypanosoma cruzi exoantigens: can those recognized by sera from chagasic patients trigger a protective immune response in mice? Res Immunol 142: 821-828.

Hoft DF, Lynch RG, Kirchhoff LV 1993. Kinetic analysis of antigenspecific immune responses in resistant and susceptible mice during infection with Trypanosoma cruzi. J Immunol 151: 7038-7047.

Kierszenbaum F, Moretti E, Sztein MB 1993. Molecular basis of Trypanosoma cruzi-induced immunosuppression. Altered expression by activated human lymphocytes of molecules which regulate antigen recognition and progression through the cell cycle. Biol Res 26: 197-207.

Minoprio P 2001. Parasite polyclonal activators: new targets for vaccination approaches? Int J Parasitol 31: 588-591.

Montes CL, Acosta-Rodríguez EV, Mucci J, Zuniga EI, Campetella O, Gruppi A 2006. A Trypanosoma cruzi antigen signals CD11b ${ }^{+}$ cells to secrete cytokines that promote polyclonal B cell proliferation and differentiation into antibody-secreting cells. Eur J Immunol 36: 1474-1485.

Parodi C, Padilla AM, Basombrío MA 2009. Protective immunity against Trypanosoma cruzi. Mem Inst Oswaldo Cruz 104 (Suppl. I): 288-294.

Rodrigues MM, de Alencar BC, Claser C, Tzelepis F, Silveira EL, Haolla FA, Dominguez MR, Vasconcelos JR 2009. Swimming against the current: genetic vaccination against Trypanosoma cruzi infection in mice. Mem Inst Oswaldo Cruz 104 (Suppl. I): 281-287.

Takehara HA, Mota I 1991. The possible mechanism of action of IgG antibodies and platelets protecting against Trypanosoma cruzi infection. Braz J Med Biol Res 24: 759-765.

Tarleton RL 1991. Regulation of immunity in Trypanosoma cruzi infection. Exp Parasitol 73: 106-109.

Umekita LF, Mota I 2000. How are antibodies involved in the protective mechanism of susceptible mice infected with T. cruzi? Braz J Med Biol Res 33: 253-258.

Vallejo GA, Guhl F, Carranza JC, Triana O, Pérez G, Ortiz PA, Marín DH, Villa LM, Suárez J, Sánchez IP, Pulido X, Rodríguez IB, Lozano LE, Urrea DA, Rivera FA, Cuba-Cuba C, Clavijo JA 2007. Trypanosoma rangeli parasite-vector-vertebrate interactions and their relationship to the systematics and epidemiology of American trypanosomiasis. Biomedica 27 (Suppl. 1): 110-118.

Vallejo GA, Guhl F, Schaub GA 2009. Triatominae-Trypanosoma cruzi/T. rangeli: vector-parasite interactions. Acta Trop 110: 137-147. 